\title{
Special Issue: Fractional calculus and its applications
}

\section{Introduction}

Scaling defines a relative measure with respect to which a model, computations or statistical analyses are made. For example, a time scale defines a measure of time, on the basis of which, models, data and statistical information are defined, recorded and analyzed. A model based on discrete time day data, defining for example a stock price from day to day differs necessarily from intraday data and of course, they differ from the theoretical definition of continuous time and data models. As a result applying fractional operators to models and data has a particular meaning. While day measurements are discrete, time recorded in a given time interval, a day, intraday measurement time intervals can vary from milliseconds to minutes and hours. Both are discrete while fractional models are continuous. They differ from classical continuous (Riemanian) models in their computational and fractional time intervals by the numerical speed of convergence that calculations are defined by. Furthermore, most complex and dynamic systems are defined over multiple time scales, contributing to systems complexity as well as to a randomness summarized by their mixtures which may call "multi-fractal". In this view, the same notion of time demands a more accurate consideration. Is the physical time appropriate to describe financial phenomena? Or could it be possible that many apparent irregularities we observe vanish under more appropriate timedeformations capable to account for the diverse intensity with whom financial events occur? The statistical implications to the many problems associated to time scales in financial modeling, in natural and social sciences are confronted raise both opportunities and challenges and a multitude of research papers and books that have approached fractional calculus and randomness from different vantage points.

The origins and the interpretation of fractional models are many and not new. There is an extensive history and developments with celebrated names such as Cauchy, Liebniz, Liouville, Abel, Caputo, Riesz and so many others that have raised questions that chal- lenged mathematicians, physicists and applied mathematicians over the last few hundreds years. There is an extensive bibliography on fractional calculus and many applications spanning physics, calculus, data analysis, stochastic and Brownian Motion, the Brownian Bridge and $\alpha$-stable distributions as they have appeared in many research areas. Doctoral theses and books have also been written and provide a broad and varied perspective to the relevance and applicability of fractional calculus.

Although there are many theoretical and applied fractional problems, it requires additional research and empirical study to assess the effects of fractional models relative to conventional (Riemanian) models. For example, consider the speed at which a train travels. A fast train that records images as it travels at high speed has relatively small informative and granular detail when compared to a "slower" moving train, that records all exists in its path. Yet, they are both observing the same landscape, each defined by the granularity of their records, that define in fact a vantage point for their analysis. Discrete time models differ from one another by the time intervals defining the resolution of the data (whether deterministic or stochastic). Each discrete time defining a snapshot of an instant which we seek to reconcile to a theoretical and granular free model (i.e. a continuous time model). Similarly, a picture taken by a pixels endowed camera may reveal far more information relative to a pixel poor camera. Fractional models, unlike discrete time models of various granularity, commonly represented by granular time series, provide therefore a continuous time interpretation of fractional models based on a parametric fractional granularity. As a result, they provide a means to reconcile theoretically the relationship between model of different granularities. Such a transformation has important implications to how information is recorded and processed as well as how fractional operators alter our measures, what we see and how we may reconcile what we see with what is.

A simple and intuitive example will highlight some of the problems and applications that fractional calcu- 
lus raises. Say that $(\Delta t)^{H}$ is a small functional time interval, used to discretize a continuous time model (when its limit is zero). Explicitly, define a discrete time model by: $\Delta S(t)=\alpha S(t)(\Delta t)^{H}, S(0)=S_{0}$. As the time limit $(\Delta t)^{H} \rightarrow 0, \Delta t \neq 1$, the differential equation $d S(t) / S(t)=\alpha(d t)^{H}, S(0)=S_{0}$ results, whose solution, when $H=1$, is trivially $S(t)=S_{0} e^{\alpha t}$. When $H \neq 1$ it differs from the solution of its discrete time equivalent $S\left(t+(\Delta t)^{H}\right)-S(t)=\alpha S(t)$, $S(0)=S_{0}$. In this case, define $d S_{H}(t)=S(t)(d t)^{H}$ and

$$
S_{H}(t)=\int_{0}^{t} S(\tau)(d \tau)^{H}, \quad 0<H<1 .
$$

Guy Jumarie [1] proves that a fractional operator (Riemann-Liouville) results:

$$
\begin{gathered}
S_{H}(t)=H \int_{0}^{t}(t-\tau)^{H-1} S(\tau) d \tau, \\
0<H<1 .
\end{gathered}
$$

Replacing $S(t)$ by a normalized Gaussian white noise $W(t)$ into the last relation yields to an alternative definition of the normalized fractional Brownian motion $B_{H}(t)$, i.e.

$$
\begin{aligned}
B_{H}(t)= & \frac{1}{\left(H+\frac{1}{2}\right) \Gamma\left(H+\frac{1}{2}\right)} \\
& \times \int_{0}^{t} W(\tau)(d \tau)^{H+1 / 2}, \quad 0<H<1,
\end{aligned}
$$

which reduces to the normalized Brownian motion in the special case $H=1 / 2$ :

$$
B(t)=B_{1 / 2}(t)=\int_{0}^{t} W(\tau) d \tau .
$$

These properties, granularity, speed of convergence (defined by the fractional parameter $(d \tau)^{H}$ calculated at the limit) and their computational issues are therefore important and have statistical, computational and analytical implications to our treatment of financial and other data models. The definition and the measurement of time and their functional granularity, thus defines the models we construct, their statistical and other properties as well as their analyses and their uses.

The frontier on these topic is challenging and wide in several directions: it ranges from the deep understanding of the very foundations of fractional calculus, to the generalizations built on a time-changing granularity, from the improvement of the computational tools, to the analysis of the scaling properties of the fractional distributions. All these the topics are to some extent discussed in the contributions of this special issue, which combines a number of applications of fractional calculus.

The Multifractional Process with Random Exponent (MPRE), a stochastic process which replaces the functional parameter of a multifractional Brownian motion by a random variable or even a stochastic process, is the focus of the contribution by Antoine Ayache, Céline Esser and Julien Hamonier, entitled "A new Multifractional Process with Random Exponent". They propose a new approach for constructing a type of MPRE different from the one previously introduced in 2005 . Starting from the stochastic integral representation of the high-frequency part of the fractional Brownian motion, their approach consists in replacing the Hurst parameter by a random variable depending on the integration variable. Among other things, the process obtained in this way offers the advantages to have a representation through classical Itô integral and to be less difficult to simulate than the first type of MPRE. Through a new methodology relying on an extensive use of the Haar basis, the Authors also study the Hölder regularity of the new MPRE, which appears to be a significantly more challenging problem than in the case of the previous one.

The aim of "Overfitting of Hurst estimators for multifractional Brownian motion: A fitting test advocating simple models", contribution by Pierre Raphaël Bertrand, Jean-Louis Combes, Marie-Eliette Dury and Doha Hadouni, is to provide simple models with a time-varying Hurst index $H$. Such models are intended to be simple as much as possible and fitted to the estimated Hurst parameter. After recalling some statistical issues pertaining the estimation of $H$, the Authors propose a fitting test for a model with a time-varying exponent. They also discuss how to select a simple model and illustrate their approach by numerous numerical simulations and an application to market finance data.

A fractional approach to the analysis of option market is also the focus of the article by Soňa Kilianová and Boris Letko, "An empirical study on using Hurst exponent estimation methods for pricing Call options by fractional Black-Scholes model". The Authors compare nine methods to estimate the Hurst index on a set of fifteen assets representing different industries and discuss the theoretical option prices deduced for each of the methods and each considered 
data length. Their analysis suggests that the fractional Black-Scholes model tends to outperform the standard Black-Scholes model.

The well recognized presence of jumps in financial records constitutes the main motivation of the article by Pierre Emmanuel Lévy dit Véhel and Jacques Lévy Véhel ("Stochastic jump intensity models"), who focus on the tempered versions of multistable processes (also known as CGMY models) and their modelling in the perspective of risk management. Multistable motions generalize Lévy motions allowing the jump intensity to vary in time in an exogenous manner. The contribution represents a first step towards a long-term program that aims at developing stochastic processes able to model all dimensions of risk in a non-stationary way. In the frame of CGMY and similar models, instantaneous variance condenses into one value influences of the four parameters C, G, M and Y, whereas - this is the point of the Authors - it is but one dimension of risk and a fine study of models such as, e.g., the Value at Risk should rather isolate the contributions of each of these parameters. Therefore, in their work the Authors study the impact on VaR of variations of the overall level of activity, as measured by $\mathrm{C}$, as well as of the intensity of jumps, as controlled by $\mathrm{Y}$.

Issue Editor, Professor Sergio Bianchi

Department of Economics and Law

University of Cassino and Southern Lazio 03043 Cassino (Italy)

\section{References}

[1] G. Jumarie, On the representation of fractional Brownian motion as an integral with respect to $(\mathrm{d} t)^{a}$, Applied Mathematics Letters 18 (2005), 739-748. doi:10.1016/j.aml.2004.05.014. 